\title{
Contribuição ao estudo de insetos (Arthropoda: Insecta) associados ao gênero Ixora L. (Angiospermae: Rubiaceae) no Brasil ${ }^{(1)}$
}

\author{
THIAGO DIAS TRINDADE ${ }^{(2)}$; LILIANE KIFFER FIGUEIRA \\ DO NASCIMENTO(3) e ANA CAROLINA MUNIZ(2)
}

\begin{abstract}
RESUMO
Esse trabalho objetivou registrar insetos (Arthropoda: Insecta) associados ao gênero Ixora L. (Angiospermae: Rubiaceae) e contribuir para novos conhecimentos acerca dessa popular planta ornamental de clima tropical no Brasil. Para realização deste trabalho foi verificada a presença de insetos em maciços ou plantas isoladas de Ixora spp., além de casas de vegetação, entre o período de 2004 e 2012. Assinalou-se pela primeira vez sobre Ixora chinensis Lam: Syntermes dirus (Burmeister, 1839) (Isoptera: Termitidae), Aspidiotus destructor Signoret, 1869 e Chrysomphalus aonidum (Linnaeus, 1758) (Hemiptera: Diaspididae), Praelongorthezia praelonga (Douglas, 1891) (Hemiptera: Ortheziidae) e Camponotus sp., (Hymenoptera: Formicidae). Registram-se pela primeira vez sobre I. coccinea L: Aleurothrixus aepim (Goeldi 1886) e Bemisia tabaci (Gennadius, 1889) (Hemiptera: Aleyrodidae), Eucalymnatus sp., Parasaissetia nigra (Nietner, 1861) e Saissetia olae (Olivier, 1791) (Hemiptera: Coccidae), e Camponotus sp.. Observou-se pela primeira vez sobre I. macrothyrsa (Teijsm. \& Binn.) N. E. Br.: Aleurothrixus floccosus (Maskell 1895) e Aleurotrachelus sp., (Aleyrodidae), Aphis (Toxoptera) citricida (Kirkaldy, 1907) e Myzus (Nectarosiphon) persicae (Sulzer, 1778) (Hemiptera: Aphididae). O presente trabalho contribui para o estudo destas importantes plantas ornamentais, apresentando pela primeira vez uma lista de insetos associados. O presente trabalho ainda cria condições para futuros estudos.Palavras-chave: planta ornamental, insetos, pragas.
\end{abstract}

\begin{abstract}
Contribution to the study of insects (Arthropoda:Insecta) associated with the genus Ixora L. (Angiospermae: Rubiaceae) in Brazil

This study aimed to register insects (Arthropoda: Insecta) associated with genus Ixora L. (Angiospermae: Rubiaceae) and contribute the knowledge about this popular tropical ornamental plant in Brazil. For this study, we detected the presence of insects in solid or isolated plants of Ixora spp., and greenhouses, between the period 2004 and 2012. It was pointed out for the first time on I. chinensis Lam.: Syntermes dirus (Burmeister, 1839) (Isoptera: Termitidae), Aspidiotus destructor (Signoret, 1869) and Chrysomphalus aonidum (Linnaeus, 1758) (Hemiptera: Diaspididae), Praelongorthezia praelonga (Douglas, 1891) (Hemiptera: Ortheziidae) and Camponotus sp. (Hymenoptera: Formicidae). Studies registered for the first time on I. coccinea L.: Aleurothrixus aepim (Goeldi, 1886) and Bemisia tabaci (Gennadius, 1889) (Hemiptera: Aleyrodidae) Eucalymnatus sp., Parasaissetia nigra (Nietner, 1861) and Saissetia olae (Olivier, 1791) (Hemiptera: Coccidae), and Camponotus sp. It was observed first on I. macrothyrsa Teijsm. \& Binn.) N. E. Br.: Aleurothrixus floccosus (Maskell, 1895), Aleurotrachelus sp. (Aleyrodidae), Aphis (Toxoptera) citricida (Kirkaldy, 1907) and Myzus (Nectarosiphon) persicae (Sulzer, 1778) (Hemiptera: Aphididae) Harmonia sp. (Coleoptera: Coccinellidae), Camponotus sp. This paper contributes to the study of these important ornamental plants, presenting for the first time a list of associated insects. This work also creates conditions for future studies. Keywords: ornamental plants, insects, pests
\end{abstract}

\section{INTRODUÇÃO}

As espécies do gênero Ixora L. (Rubiaceae) são originárias da Ásia (LORENZI e SOUZA, 2001) e se encontram amplamente distribuídas no Brasil, em ambientes domésticos ou públicos, sendo utilizadas como maciços ou arbustos isolados. São conhecidas pelo florescimento constante e a tolerância à falta d'água e a pragas, razões que levam ao largo uso em canteiros ajardinados e praças públicas, sobretudo na região sudeste do país, cujo clima quente inviabiliza o cultivo de outras espécies mais exigentes.

Trindade (2011) apresentou como novo hospedeiro de C. viridis, I. macrothyrsa (Teijsm. \& Binn.) N. E. Br., registrando-se também para esta espécie botânica as moscasbrancas Aleurothrixus aepim (Goeldi, 1886), Bemisia tabaci (Gennadius, 1889) e Minutaleyrodes minuta (Singh, 1931) (Hemiptera: Aleyrodidae), e o predador Chrysoperla sp. (Neuroptera: Chrysopidae). Ainda citando Trindade (2011), assinalamos outros municípios do estado do Rio de Janeiro que apresentaram a ocorrência da cochonilha-verde sobre espécies do gênero Ixora: Itaguai (I. macrothyrsa), Niterói (I. coccinea) e Rio de Janeiro (I. coccinea).

Esse trabalho objetivou registrar insetos associados ao gênero Ixora e contribuir para novos conhecimentos acerca dessa popular planta ornamental de clima tropical no Brasil.

\footnotetext{
(1) Trabalho recebido para publicação em 29/11/2014 e aprovado em 26/03/2015

(2) Universidade Federal Rural do Rio de Janeiro (UFRRJ), CTUR, Seropédica-RJ, Brasil. *Autor correspondente: thiagotdt@hotmail.com

(3) Universidade Federal Rural do Rio de Janeiro (UFRRJ), Seropédica-RJ, Brasil.
} 


\section{MATERIAL E MÉTODOS}

Para realização deste trabalho foi verificada a presença de insetos em maciços ou plantas isoladas de Ixora sp., além de casas de vegetação, entre o período de 2004 e 2012. Os insetos foram acondicionados em envelopes de papel e lacrados, ou em tubetes plásticos com álcool $70 \%$ e encaminhados para o laboratório da Coleção Entomológica Ângelo Moreira da Costa Lima (CECL), na Universidade Federal Rural do Rio de Janeiro, SeropédicaRJ. De acordo com cada espécime coletado, foi realizada montagem específica e o material coletado foi identificado com auxílio de microscópios estereoscópicos Wild M 5 e Olympus SZ 40 e microscópios Wild M 20 e Olympus BX 41 e os trabalhos de Hempel (1922a e b), Bondar (1923 e 1928), Costa Lima (1928), Blackman e Eastop (1984), Freitas (2003), Silva et al. (2004), Martin (2004, 2005 e 2008), Evans (2008) e Trindade (2011).

O material foi depositado na Coleção Entomológica Ângelo Moreira de Costa Lima (CECL).

\section{RESULTADOS E DISCUSSÃO}

Assinalaram-se quatro ordens de insetos, distribuídos em oito famílias, quinze gêneros e dezesseis espécies. Este trabalho assinalou três estados distribuídos em quinze municípios.

\section{Coleoptera:}

Coccinellidae

Harmonia sp.:

I. macrothyrsa: 11.vii.2011, em Nilópolis-RJ (Simões).

\section{Hemiptera-Sternorrhyncha:}

\section{A. Aleyrodidae}

Aleurothrixus aepim (Goeldi, 1886)

I. macrothyrsa: 11.vii.2011, em Nilópolis-RJ (Simões).

I. coccinea: 01.viii.2010, em Belford Roxo-RJ

(Trindade); 22.iv.2012, em Mesquita-RJ (Amaral).

Aleurothrixus floccosus Maskell, 1895

I. macrothyrsa: 06.viii.2011, em Nilópolis- RJ (Simões). Aleurotrachelus sp.

I.macrothyrsa: 06.viii.2011, em Nilópolis (Simões)

Bemisia tabaci (Gennadius, 1889)

I. coccinea: 10.vi.2008, em Campinas-SP (Silva); 06.xi.2008, em Vassouras-RJ (Nascimento); 18.iv.2012, no Rio de Janeiro-RJ (Trindade).

I. macrothyrsa: 27.viii.2012, no Rio de Janeiro-RJ (Trindade).

\section{B. Aphididae}

Aphis (Toxoptera) citricida (Kirkaldy, 1907)

I. macrothyrsa: 31.vii.2012, no Rio de Janeiro-RJ (Trindade).
Myzus (Nectarosiphon) persicae (Sulzer, 1776)

I. macrothyrsa: 13.iii.2007, em Nova Iguaçu-RJ (Trindade); 09.ix.2010, no Rio de Janeiro-RJ (Freitas)

\section{Coccidae}

Coccus viridis (Green, 1889)

I. chinensis: 21.iv.2007, Seropédica-RJ (Trindade); 29.iv.2010, no Rio de Janeiro-RJ (Trindade); 05.viii.2009, em São Paulo-SP (Silva); 13.iii.2012, em Guaratinguetá-SP (Santos)

I. coccinea: 15.ix.2006, no Rio de Janeiro-RJ (Trindade); 01.iii.2011, em Mendes-RJ (Trindade); 18.v.2011, em Vitória-ES (Cassiano); 20.v.2011, em Vila Velha-ES (Cassiano)

I. macrothyrsa: 25.vii.2011, em São Paulo-SP (Lima); 07.v.2012, em Campinas-SP (Silva); 14.vi.2012, em Nilópolis-RJ (Wanderley); 02.ix.2012, em Mesquita-RJ (Wanderley)

Eucalymnatus sp.

I.coccinea: 20.vii.2008 em Paracambi-RJ (Ferreira Junior)

Parasaissetia nigra (Nietner, 1861)

I. coccinea: 13.vi.2005, em Nova Iguaçu-RJ (Trindade); 09.iv.2010, no Rio de Janeiro-RJ (França)

Saissetia olae (Olivier, 1791)

I. coccinea: 31.vii.2010, em Nova Iguaçu-RJ (Trindade); 13.v.2012, em Campinas-SP (Silva)

D. Diaspididae

Aspidiotus destructor (Signoret, 1869)

I. chinensis: 17.v.2011, no Rio de Janeiro-RJ (Silva); 25.v.2011, em Campos-RJ (Ferreira); 02.ix.2012, em Nova Iguaçu-RJ (Alencar)

Chrysomphalus aonidum (Linnaeus, 1758)

I. chinensis: 10.iii.2009, em Nova Iguaçu-RJ (Trindade)

E. Ortheziidae

Praelongorthezia praelonga (Douglas, 1891)

I. chinensis: 06.vii.2011, em Vitória-ES (Cassiano); 12.ix.2011, no Rio de Janeiro-RJ (Trindade)

\section{Hymenoptera}

Formicidae

Camponotus sp.

I. chinensis: interagindo com P. praelonga, em VitóriaES (Cassiano), no Rio de Janeiro (Trindade).

I. coccinea: interagindo com $C$. viridis, em MendesRJ (Trindade), Vitória-ES (Cassiano), Vila Velha-ES (Cassiano); B. tabaci (Trindade) e P. nigra (França), no Rio de Janeiro;

I. macrothyrsa: interagindo com C. viridis, em São Paulo-SP (Lima), em Campinas-SP (Silva) 


\section{Isoptera}

Termitidae

Syntermes dirus Burmeister, 1839

I. chinensis: $15 . i v .2004$, em Nova Iguaçu-RJ (Trindade)

Assinalou-se pela primeira vez sobre I. chinensis: $A$. destructor, Camponotus sp., C. aonidum, P. praelonga e $S$. dirus.

Registram-se pela primeira vez sobre I. coccinea: $A$. aepim, B. tabaci, Camponotus sp., Eucalymnatus sp., $P$. nigra e $S$. olae.

Observou-se pela primeira vez sobre I. macrotyrsa: $A$. floccosus, Aleurotrachelus sp, Camponotus sp., Harmonia sp., M. (Nectarosiphon) persicae e A. (Toxoptera) citricida.

Trindade (2011) observou, em I. macrothyrsa, a interação entre C. viridis, A. aepim, B. tabaci e M. minuta e neste trabalho acrescenta-se este assinalamento entre Aleurotrachelus sp. e A. floccosus.

Acrescenta-se ao trabalho de Trindade e Lima (2012) a preferência de $M$. (Nectarosiphon) persicae e A. (Toxoptera) citricida por brotações novas de I. macrothyrsa.

Foi observada a presença de Capnodium spp. (Capnodiaceae) mais conhecido como fumagina, em, I. macrothyrsa (Nilópolis, Rio de Janeiro, Seropédica, Santos) e $I$. coccinea (Itu). Os danos decorrentes da penetração do estilete dos insetos fitossugadores podem acarretar hipertrofia das células cambiais e eventual colapso das células do floema, em vista da inoculação de toxinas. Os fotoassimilados transportados pelo floema se tornam comprometidos e todo o metabolismo da planta é alterado, provocando definhamento da planta e queda das folhas. Como a capacidade fotossintética da planta foi afetada por conta das fezes adocicadas serem substrato de desenvolvimento de fungos do gênero Capnodium Mont., há redução no desenvolvimento e produtividade, levando a perda na produção (KINDLER et al., 1973; HOWER 1989; HUTCHINS e PEDIGO 1989; MAU e KESSING, 1992; GALLO et al., 2002; MOREIRA et al., 2007; TRINDADE, 2011). As fezes adocicadas desses fitossugadores atraem formigas doceiras, como Camponotus sp. que delas se alimentam e como contrapartida oferecem proteção a esses insetos contra eventuais predadores. Dessa forma a população de fitossugadores aumenta sobre a planta (TRINDADE et al., 2011). Possivelmente outras espécies de formiga se valem da alimentação dessas fezes adocicadas, sobre Ixora sp.

Em Nova Iguaçu foi assinalado que sob maciço de I. chinensis havia murundu de S. dirus. Notadamente a presença deste cupim, cuja característica é o forrageamento de celulose morta, não levando prejuízo às plantas. Registrou-se pela primeira vez a associação deste cupim com essa espécie ornamental.

O coccinelídeo Harmonia sp. foi observado predando A. aepim (em I. macrothyrsa, em Nilópolis).

As plantas observadas em canteiros de calçadas e praças públicas apresentaram muitos danos mecânicos em decorrência de variadas formas de vandalismo, porém alta capacidade de recuperação de massa verde.
Em termos gerais, o gênero Ixora apresenta alto grau de tolerância à infestação de pragas, chegando a produzir razoável quantidade de flores, mesmo que tomadas por insetos, no caso, $C$. viridis.

Como essas plantas têm pouco ou nenhum manejo fitossanitário competente (em alguns lugares, populares realizam podas de controle de volume da massa verde, deixando as pontas cortadas sobre o solo do canteiro), estas podem servir como potenciais disseminadores de pragas para outras plantas, que eventualmente sendo suscetíveis podem vir a causar transtornos a transeuntes e a bens patrimoniais.

Consideramos ainda que A. aepim, A. floccosus, B. tabaci, C. viridis, C. aonidum, P. praelonga, S. olae, M. persicae, A. (Toxoptera) citricida possuem ampla distribuição mundial e são associados a culturas de grande importância econômica, sendo ainda polífagas e capazes de transmitir uma série de viroses fitopatogênicas.

\section{CONCLUSÕES}

O presente trabalho contribui para o estudo desta importante planta ornamental, apresentando pela primeira vez uma lista de insetos associados. O presente trabalho ainda cria condições para futuros estudos.

\section{REFERÊNCIAS}

BLACKMAN, R.L.; EASTOP, V.F. Aphids on the world's crops: an identification guide. Chichester: J. Wiley, 1984. $466 \mathrm{p}$.

BONDAR, G. Aleyrodídeos do Brasil: catálogo descriptivo dos Hemípteros-Homópteros da família dos Aleyrodídeos, insectos parasitas das plantas, encontrados no Brasil. [Salvador]: Laboratório de Pathologia Vegetal do Estado da Bahia, Secretaria da Agricultura, Indústria e Obras Públicas do Estado da Bahia, 1923. 183p.

BONDAR, G. Aleyrodideos do Brazil (2a contribuição). Boletim do Laboratório de Pathologia Vegetal do Estado da Bahia, Salvador, v.5, p.1-37, 1928.

COSTA LIMA, A.M. DA. Contribuição ao estudo dos aleyrodideos da subfamília Aleurodicinae. Memórias do Instituto Oswaldo Cruz, Rio de Janeiro, v.4, suplemento, p.128-140, 1928.

EVANS, G. 2008. The whiteflies (Hemiptera: Aleyrodidae) of the world and their host plants and natural enemies. Version 2008-09-23. Disponível em $<$ http://keys.lucidcentral.org/keys/v3/whitefly/PDF PwP\%20ETC/world-whitefly-catalog-Evans.pdf $>$. Acesso em 06 de Junho de 2014.

FREITAS, S. Chrysoperla Steinmann, 1964 (Neuroptera, Chrysopidae): descrição de uma nova espécie do Brasil. Revista Brasileira de Entomologia, Curitiba, v.47, n.3, p.385-387, 2003. 
GALLO, D.; NAKANO, O.; SILVEIRA NETO, S., CARVALHO, R.P.L.; BAPTISTA, G.C. DE; BERTI FILHO, E.; PARRA, J.R.P.; ZUCCHI, R.A.; ALVES, S.B.; VENDRAMIM, J.D.; MARCHINI, L.C.; LOPES, J.R.S.; OMOTO, C. Entomologia Agrícola. Piracicaba: Fundação de Estudos Agrários Luiz de Queiroz, Biblioteca de Ciências Agrárias Luiz de Queiroz, v.10, 2002. 920p.

HEMPEL, A. Algumas espécies novas de Hemipteros da família Aleyrodidae. Notas Preliminares editadas pela redacção do Museu Paulista, São Paulo, v.2, p.3-10, 1922a.

HEMPEL, A. Hemipteros novos ou pouco conhecidos da família Aleyrodidae. Revista do Museu Paulista, São Paulo, v.13, p.1121-1191, 1922b.

HOWER, A.A. Potato leafhopper as a plant stress factor on alfalfa, p.35-39. In: ARMBRUST, E.J.; LAMP, W.O. (Eds.). History and perspectives of potato leafhopper (Homoptera: Cicadellidae) research. Miscellaneous Publication of the Entomological Society of America, Lanham, v.72, p.1226, 1989.

HUTCHINS, S.H.; PEDIGO, L.P. Potato leafhopper induced injury on growth and development of alfalfa. Crop Science, Madison, v.29, n.4, p.1005-1011, 1989. DOI: $<$ http://dx.doi.org/10.2135/cropsci1989.0011183X002900 $040036 \mathrm{x}>$

KINDLER, S.D.; KEHR, W.R.; OGDEN, R.L.; SHALK, J.M. Effect of potato leafhopper injury on yield and quality of resistant and susceptible alfalfa clones. Journal of Economic Entomology, Annapolis, v.66, n.6, p.12981302, 1973.

LORENZI, H.; SOUZA, H.M. Plantas ornamentais no Brasil: arbustivas, herbáceas e trepadeiras, $3 \mathrm{ed}$. Nova Odessa: Instituto Plantarum de Estudos da Flora Ltda., 2001. 1130p.

MAU, R.F.L.; KESSING, J.L.M. 1992. Anoplolepis longipes (Jerdon). Disponível em <http://www.extento. hawaii.edu/kbase/crop/type/A_longip.htm>. Acesso em: 13 de janeiro de 2012 .
MARTIN, J.H. The whiteflies of Belize (Hemiptera: Aleyrodidae) Part 1 - Introduction and account of the subfamily Aleurodicinae Quaintance \& Baker. Zootaxa, Auckland, n.681, p.1-119, 2004.

MARTIN, J.H. The whiteflies of Belize (Hemiptera: Aleyrodidae) Part 2 - A review of the subfamily Aleyrodinae Westwood. Zootaxa, Auckland, n.1098, p.1-100, 2005.

MARTIN, J.H. A revision of Aleurodicus Douglas (Sternorrhyncha, Aleyrodidae), with two new genera proposed for palaeotropical natives and an identification guide to world genera of Aleurodicinae. Zootaxa, Auckland, n.1835, p.1-100, 2008.

MOREIRA, M.D.; FERNANDES, F.L.; PICANÇO, M.C.; FERNANDES, M.E.S.; BACCI, L.; MARTINS, J.C.; COUTINHO, D.C. Características rastreáveis do manejo integrado das pragas do cafeeiro. In: ZAMBOLIM, L. (Ed.). Rastreabilidade para a cadeia produtiva do café. Viçosa: UFV, 2007. 450p.

SILVA, R.A. DA; MICHELOTTO, M.D.; JORDÃO, A.L. Levantamento preliminar de pulgões no estado do Amapá. Circular Técnica, Embrapa Amapá, Macapá, n.32, p.111, 2004.

TRINDADE, T.D.; LIMA, A.F.; RACCA FILHO, F. Espécies de Paraleyrodes Quaintance (Hemiptera: Aleyrodidae) no estado do Rio de Janeiro, Brasil. Entomotropica, Maracay, v.26, n.2, p.69-77, 2011.

TRINDADE, T.D. Contribuição sobre distribuição e novos hospedeiros de Coccus viridis Green, 1889 (Hemiptera: Coccidae) no estado do Rio de Janeiro, Brasil. Entomotropica, Maracay, v.26, n.3, p.147-152, 2011.

TRINDADE, T.D.; LIMA, A.F. Predação de espécies de moscas brancas (Hemiptera: Aleyrodidae) por Chrysoperla Steinmann (Neuroptera: Chrysopidae) no estado do Rio de Janeiro - Brasil. Entomotropica, Maracay, v.27, n.2, p.1$27,2012$. 